\title{
Characterising young visual M-dwarf binaries with near-infrared integral field spectra ${ }^{\star, \star \star}$
}

\author{
Per Calissendorff' ${ }^{1}$, Markus Janson ${ }^{1}$, and Mickaël Bonnefoy ${ }^{2}$ \\ 1 Department of Astronomy, Stockholm University, Stockholm, Sweden \\ e-mail: per.calissendorff@astro.su.se \\ 2 Univ. Grenoble Alpes, CNRS, IPAG, 38000 Grenoble, France
}

Received 26 March 2020 / Accepted 21 July 2020

\begin{abstract}
We present the results from an integral field spectroscopy study of seven close visual binary pairs of young M-dwarf multiple systems. The target systems are part of the astrometric monitoring AstraLux programme, surveying hundreds of M-dwarf systems for multiplicity and obtaining astrometric epochs for orbital constraints. Our new VLT/SINFONI data provides resolved spectral type classifications in the $J, H$, and $K$ bands for seven of these low-mass M-dwarf binaries, which we determine by comparing them to empirical templates and examining the strength of water absorption in the $K$ band. The medium resolution $K$-band spectra also allows us to derive effective temperatures for the individual components. All targets in the survey display several signs of youth, and some have kinematics similar to young moving groups, or low surface gravities which we determined from measuring equivalent widths of gravity sensitive alkali lines in the $J$ band. Resolved photometry from our targets is also compared with isochrones from theoretical evolutionary models, further implying young ages. Dynamical masses will be provided from continued monitoring of these systems, which can be seen as emblematic binary benchmarks that may be used to calibrate evolutionary models for low-mass stars in the future.
\end{abstract}

Key words. binaries: close - binaries: visual - stars: fundamental parameters - stars: low-mass - techniques: spectroscopic

\section{Introduction}

Binaries and higher order hierarchical multiple systems of stars provide a plethora of useful information when studied. Population properties can provide insight to the different formation pathways and evolution, connecting higher mass stars to their lower mass counterparts and to objects at the very low-mass bottom tail of the initial mass function, such as brown dwarfs. Keplerian motions in multiple systems reveal dynamical masses of the components, which together with their other physical features including age and brightness can be used to calibrate massluminosity relations (e.g. Burgasser et al. 2007; Goodwin et al. 2008; Janson et al. 2007; Köhler et al. 2012; Duchêne \& Kraus 2013). The more precisely these characteristics can be constrained, the better our physical interpretation becomes, forming some of our most fundamental understanding of stellar properties. Even though mid- to late-type M dwarfs are the most numerous of the stellar types in the solar neighbourhood, the fundamental physical characteristics such as age, mass, luminosity, radius, and their relations are poorly constrained compared to intermediate-mass and solar-type stars. Since multiplicity frequency decreases for later spectral types and lower primary mass, suitable benchmark multiple systems with detailed studies of orbital elements, and thereby dynamical masses, are lacking for low-mass $\mathrm{M}$ dwarfs. As such systems are required for calibrating theoretical evolutionary models, significant effort

\footnotetext{
* The reduced spectra are only available at the CDS via anonymous ftp to cdsarc.u-strasbg.fr (130.79.128.5) or via http: //cdsarc.u-strasbg.fr/viz-bin/cat/J/A+A/642/A57

$\star \star$ Based on observations made with ESO telescopes at the Paranal Observatory under programme ID 0102.C-0186.
}

has been made in recent years to enhance the characterisation of cool dwarfs, for mid- to late $\mathrm{M}$ types and for substellar objects (e.g. Bouy et al. 2008; Bonnefoy et al. 2009, 2014; Dupuy et al. 2010; Schlieder et al. 2014; Calissendorff et al. 2017, 2019; Calissendorff \& Janson 2018). Furthermore, with the recent abundant discoveries of planets with M-star hosts (e.g. Dressing \& Charbonneau 2013; Gillon et al. 2016), accurate stellar physical properties are required in order to derive reliable parameters for properties of the planetary systems, advancing the appeal to better characterise $M$ dwarfs (e.g. Johnson et al. 2012; Muirhead et al. 2012, 2014; Gaidos et al. 2014; Bowler et al. 2015).

For young M dwarfs, typically below $\leq 100 \mathrm{Myr}$, atmospheric and evolutionary models are particularly poorly constrained since only a handful of young binaries have had their dynamical masses measured (e.g. Close et al. 2005; Bonnefoy et al. 2009; Montet et al. 2015; Mizuki et al. 2018; Rodet et al. 2018). However, one of the most difficult quantities to fully constrain is the age of a given system, which tends to dominate the uncertainty for most cases. Therefore, it becomes essential to identify systems where ages can be more easily assessed, such as associating them with kinematic groups with known ages, i.e. young moving groups (YMGs), which are expected to be largely coeval (e.g. Zuckerman \& Song 2004; Torres et al. 2008). Due to the coevality assumption of these systems, they can be subjected to more robust population age determination methods than is possible for individual stars.

The goal of the AstraLux large $M$ dwarf survey is to improve constraints on multiplicity properties for $\mathrm{M}$ dwarfs and to identify young binaries that are suitable for orbital monitoring so that dynamical mass measurements can be carried out 
Table 1. Log of VLT/SINFONI observations.

\begin{tabular}{|c|c|c|c|c|c|c|}
\hline 2MASS ID & Alt. name & Obs. date & $\begin{array}{c}\text { Exp. time } \\
J\end{array}$ & $\begin{array}{l}\text { Exp. time } \\
H+K\end{array}$ & Airmass & $\begin{array}{c}\text { Telluric STD } \\
\text { SpT }\end{array}$ \\
\hline $1112542+1526214$ & GJ 30 & 18 & $5 \times 20 s$ & $5 \times 2 s$ & $\approx 1.4$ & B5 \\
\hline 2MASS J03323578+2843554 & UCAC4 594-08941 & $27-N o v-2018$ & $5 \times 30 \mathrm{~s}$ & $5 \times 30 \mathrm{~s}$ & $\approx 1.9$ & B2 \\
\hline 2MASS J04595855-0333123 & UCAC4 433-008289 & 4-Nov-2018 & $5 \times 30 \mathrm{~s}$ & $5 \times 30 \mathrm{~s}$ & $\lesssim 1.2$ & B7 \\
\hline 2MASS J06112997-7213388 & AL 442 & 28-Nov-2018 & $5 \times 30 \mathrm{~s}$ & $5 \times 30 \mathrm{~s}$ & $\lesssim 1.5$ & B4 \\
\hline 2MASS J10140807-7636327 & K2001c 27 & 24-Nov-2018 & $5 \times 30 \mathrm{~s}$ & $5 \times 30 s$ & $\approx 1.7$ & B9 \\
\hline 2MASS J10364483+1521394 & UCAC4 527-051290 & 26-Feb-2019 & $5 \times 30 \mathrm{~s}$ & $5 \times 10 \mathrm{~s}$ & $\approx 1.3$ & B4, B5 \\
\hline 2MASS J23495365+2427493 & UCAC4 573-135909 & 9-Jun-2019 & $5 \times 30 \mathrm{~s}$ & $5 \times 30 \mathrm{~s}$ & $\approx 1.7$ & $\mathrm{~B} 2, \mathrm{~B} 5$ \\
\hline
\end{tabular}

(Janson et al. 2014a,b). By employing Lucky Imaging, the multiplicity survey discovered 761 nearby late $\mathrm{K}$ and $\mathrm{M}$ dwarfs (Bergfors et al. 2010; Janson et al. 2012), with an extension of the survey bringing 286 more later-type M dwarfs (Janson et al. 2014a). Recent studies of kinematics of YMGs and associations have revealed that a large fraction of the AstraLux targets are high-probability members of these young associations. To better constrain the spectral types and youth of some of these M dwarfs, we selected seven binary pairs or triple systems from the survey, five of which have strong indications of being members of YMGs, which were picked for integral field spectroscopic observations that are presented in this paper. These seven systems are all close visual binaries, whose observed properties including luminosity, spectral type, and surface gravity can be compared to theoretical evolutionary models of pre-main sequence stars, which in turn yield mass for individually resolved components and age estimates. As the systems are part of an ongoing astrometric monitoring survey, orbital constraints and thereby dynamical masses will be determined for these objects within a few years, and can be compared to the results presented here.

This paper is outlined as follows after the introduction in Sect. 1. Target description with observations and data reduction are described in Sect. 2. In Sect. 3 we present the methods applied for individual component spectral type characterisation and effective temperature measurements, as well as the procedure for measuring equivalent widths (EWs) of gravity sensitive features, which are then compared to old field stars and very young objects. In Sect. 4 we discuss our results on individual systems, correlating different youth indicators and comparing measured mass-luminosity relations. A summary and conclusions from this study are presented in Sect. 5.

\section{Observations and data reduction}

\subsection{Sample selection and observations}

The observed sample consists of seven nearby M-dwarf binaries or higher hierarchical triple systems with a closely separated binary pair. These systems were chosen from the AstraLux M dwarf survey target list and were deemed good candidates for follow-up observations with near-infrared spectroscopy and characterisation, similar to the target list composed by Bergfors et al. (2016). The targets are listed in Table 1 where names are otherwise abbreviated from the full Two Micron AllSky Survey (2MASS) identifier to the form Jhhmm. All targets were observed with Lucky Imaging by AstraLux over several epochs to probe common proper motion and are confirmed as physically bound, bona fide binaries. The binaries all have projected separations of $a \leq 9 \mathrm{AU}$ and are within distances $d \leq$ $60 \mathrm{pc}$ of the Sun. Furthermore, all targets are expected to be young based on their activity, YMG membership, or low surface gravity measurements, most of them with expected ages ranging from 20-40 Myr, with the exception of J1036 which has been suggested to be part of the $\sim 400$ Myr Ursa Majoris moving group (Mamajek et al. 2010; Jones et al. 2015). Four of our targets, J0459, J0611, J1036, and J2349 had their parallax and proper motions measured by Gaia DR2 (Gaia Collaboration 2016, 2018), but no data for J0111, J0332, or J1014 exist in the current release.

Based on the known kinematics of individual systems and using the Banyan $\Sigma$-tool (Gagné \& Faherty 2018) for estimating YMG membership probabilities, we find J0111 and J0332 to be part of the $\approx 24 \mathrm{Myr}$ old $\beta$ pic moving group with $99.7 \%$ and $97 \%$ probabilities, respectively. Previous estimates for the J0459 system with the BANYAN II (Gagné et al. 2014a) suggested it was part of the $\approx 40$ Myr Argus moving group, but the newer BANYAN $\Sigma$-tool with updated Gaia DR2 proper motions and parallax finds that the system is part of the field population with a $99.9 \%$ probability. We find that J0611 (95\% probability) and J1014 (92.5\% probability) are both part of the $\approx 45 \mathrm{Myr}$ old Carina moving group. However, the distance to the J1014 system is somewhat clouded in ambiguity. Malo et al. (2013) suggests a statistical distance to the $\mathrm{J} 1014$ system of $69 \mathrm{pc}$ based on its moving group membership, whereas Riaz et al. (2006) finds a closer spectroscopic distance of $14 \mathrm{pc}$. Neither distance estimate has any affect on the BANYAN $\Sigma$ YMG membership probability. J1036 is a triple system with an equal brightness outer binary, and was previously assigned to the $\approx 400$ Myr Ursa Majoris moving group (Klutsch et al. 2014), but more recent kinematic measurements by Gaia DR2 and using the BANYAN $\Sigma$-tool suggests that the system is more likely to belong to the field (99.9\% probability). Previous YMG membership probabilities from using the BANYAN II tool suggested the J2349 system to be a likely member of the $\beta$ pic or Columba moving groups $(94.8 \%$ and $5.1 \%$ probability, respectively) (Janson et al. 2014b). Using the BANYAN $\Sigma$-tool on the other hand, we find that the system belongs to the field ( $99 \%$ probability).

Observations were carried out in service mode with the Adaptive Optics fed Spectrograph for INtegral Field Observations in the Near Infrared ${ }^{1}$ (SINFONI; Eisenhauer et al. 2003) at the Very Large Telescope Unit 4 (Yepun). The SINFONI instrument consists of the Spectrograph for Infrared Faint Field Imaging (SPIFFI) that is being fed by a modified version of the MultiApplication Curvature Adaptive Opticts system (MACAO Bonnet et al. 2003). The observations were part of the ESO 0102.C-0186(A) programme and executed between November 2018 to June 2019. Exact dates for the observations of individual targets and their respective exposure times for each band

\footnotetext{
1 SINFONI has since been decommissioned.
} 
are listed in Table 1. All targets were observed with the $J$ $(\lambda=1.1-1.4 \mu \mathrm{m})$ and $H+K(\lambda=1.45-2.45 \mu \mathrm{m})$ gratings, which have resolving powers of $R \approx 2000$ and $R \approx 1500$, respectively. The targets were sufficiently bright themselves and acted as natural guide stars. For most targets the narrow field mode was employed, providing a spaxel scale of 12.5 mas $\times 25$ mas and a field of view of $0.8 \operatorname{arcsec}^{2}$, with the only exception being J0111, whose binary separation was too large and consequently observed with the larger $8 \operatorname{arcsec}^{2}$ field of view and 125 mas $\times 250$ mas spaxel scale. Each source was observed with a small dithering pattern of five different positions in order to correct for bad pixels and to encompass all components, including the primary components for the triplet systems, J0332 and J1036. A skyframe was taken following the science observations, mimicking the exposure time of the science frame. The airmass ranged between 1.1 and 1.9 for separate targets, and each observation was accompanied by observations of a telluric standard star of spectral type B with similar airmass. Separate telluric standard stars were observed for J1036 for each band observed in, with a B4 spectral type for $J$ band and a B5 spectral type for $H+K$ band. Additionally, two telluric standard stars were observed together with $\mathrm{J} 2349$ because the first standard star is a resolved visual binary itself, thus diluting the point spread function (PSF).

\subsection{Data reduction}

Raw data and associated calibration frames were compiled to construct $J$ - and $H+K$-band datacubes using the ESO reduction pipeline Esoreflex (Freudling et al. 2013) for SINFONI, where all frames were combined to a final co-added datacube. An empirical polynomial law was applied to the data to correct for the target shift with wavelength induced by the atmospheric refraction. The quality of the data was inspected by eye, where we noted that for J2349 only the first frame was usable, and the component positions were either outside or partly outside the field of view in the remaining frames, causing a streak of lowsignal pixels to move through the secondary component. Thus, only the datacube from the first frame for J2349 was used for further analysis.

All components in the resulting final datacubes were resolved with sufficient signal-to-noise ratio that individual spectral elements could be extracted. Nevertheless, for these close binaries cross-contamination is an issue, which we addressed by applying the same procedure as presented in Calissendorff et al. (2019), where a custom spectral extraction script is made to deblend the flux of the components for each wavelength slice within the datacube. The script is based on a grid-search in $x$ and $y$ positions (right ascension and declination) in the field of view, where a bright standard star is used as a PSF reference to find the optimal positions, and simultaneously scaled in brightness to match the respective component. We then subtract the PSF reference from one of the component positions and clean the image by removing the halo with a smoothed Gaussian profile fitted to the remaining binary component. The procedure is then repeated for the second component to minimise cross-contamination. A model system is then produced where the PSF reference is placed at the positions for the components and scaled to match their respective brightness, which is successively subtracted from the original frame to procure a residual image that can be compared to other positions and brightness scaling to find the optimal grid parameters.

Since the PSF values at the time of the observations of the targets were not necessarily the same as the values of the stan- dard star observed just before or after, we considered a second approach as well where we constructed a PSF reference by duplicating the brightest component in the target system, similar to what is done by Bergfors et al. (2016). We adopt the same naming of the two different approaches as Bergfors et al. (2016), calling the standard star reference PSF std and the duplicated primary component reference PSFdup. For all considered cases, the PSFdup reference achieved the smallest residuals from our PSF matching algorithm for spectral extraction, and therefore considered more accurate. We thus performed all following calculations on the data sets where the PSFdup reference was applied. Nevertheless, given the proximity to the secondary component for some systems, the PSFdup reference may contain higher contamination during the deblending process described above.

Additionally, we separated the $H$ and $K$ bands by only considering slices in the wavelength span of $\lambda=1.45-1.80 \mu \mathrm{m}$ for the $H$ band and $\lambda=1.945-2.415 \mu \mathrm{m}$ for the $K$ band. We also pruned the $J$ band to wavelengths $\lambda=1.10-1.35 \mu \mathrm{m}$. The choice of wavelength ranges was made in order to minimise inclusion of strong telluric water regions and to remove the edges of the passbands where SINFONI performs poorly.

The procedure is sensitive to how closely the binary components are separated, especially in the PSFdup scenario when one component is acting as the reference for the other. For certain cases (e.g. J1036) the deblending process may be less accurate when one component is close to the edge of the detector and the entire halo is not within the field of view.

Circular aperture photometry was performed for both science targets and telluric standards to obtain spectra, where aperture sizes were kept the same for targets as for their respective standard stars to minimise differential flux losses. Typical aperture size was set to $\approx 10-20$ spaxels in radius, centred upon the photocentre of the binaries in order to obtain unresolved fluxes. The size of aperture radius was decided depending on the projected separation between the binary components for each individual system, with a greater aperture size for a larger separation. Thus, undesired noise may enter the aperture when the separation and thereby the aperture was large, contributing to the uncertainty on our spectral analysis. The unresolved flux was thereafter scaled according to the brightness scaling parameter derived from the PSF spectral extraction tool described above. The telluric standard star spectra were then cleaned from strong absorption lines, including the $\mathrm{Pa} \beta$ line in the $J$ band and the $\mathrm{Br}$-series in the $H+K$ band. We first divided the standard star spectra by a transmission spectrum from the ESO sky model toolSkyCalc ${ }^{2}$, and divided the resulting standard star spectra by a blackbody function of the corresponding temperature for the standard star. The resulting spectral response is then a flat spectrum containing mainly stellar and instrumental features. We sequentially fit a Gaussian profile to each strong spectral feature, and interpolate the continuum from either side of the feature over it, starting from a full width half maximum length outwards from its centre. The previous steps simplify the Gaussian fitting procedure, as the spectra are flat and free from strong telluric features at wavelengths near the hydrogen lines of interest. The spectra are then again multiplied by the sky model transmission spectrum, resulting in a stellar absorption free spectral response by which the observed binary spectra are thereafter divided to obtain our final science spectra.

2 http://www. eso.org/observing/etc/bin/gen/form?INS. MODE=Swspectr+INS . NAME=SKYCALC 


\section{Methods}

\subsection{Spectral type}

The continuum of near-infrared spectra is sensitive to temperature changes, and also possesses strong atomic and molecular line features, and is therefore useful for probing spectral types of cool stars such as those in our sample. We follow the spectral best-fit procedure described in Cushing et al. (2008) to derive continuum spectral types of our resolved binary components, comparing them to empirical template spectra of cool M dwarfs from the NASA Infrared Telescope Facility (IRTF) Spectral Library (Cushing et al. 2005; Rayner et al. 2009) and younger late $\mathrm{M}$ types and $\mathrm{L}$ types from the Montreal Spectral Library (Gagné et al. 2015). The empirical templates used for comparison are listed in Table 2. The observed spectra are first resampled to match the resolution of the template, and the goodness-of-fit value, $G$, is calculated accordingly as

$G=\sum_{i=1}^{n}\left(\frac{f_{i}-C F_{i}}{\sigma_{i}}\right)^{2}$

for each individual model, where $n$ is the number of data points, or wavelength slices in this case, with $i$ being the wavelength index; $f_{i}$ and $F_{i}$ are the observed and model flux, respectively, with $\sigma_{i}$ being the uncertainty; and $C$ is a scaling factor calculated as

$C=\frac{\sum f_{i} F_{i} / \sigma_{i}^{2}}{\sum F_{i}^{2} / \sigma_{i}^{2}}$.

For our continuum shape spectral analysis, we assign the template with the lowest calculated $G$ value as the best fit. We do this separately for all three bands considered, giving the resulting best-fit spectral type in Table 3, and show all binary spectra together with the IRTF models for each respective band in Figs. 1-3, where the observed spectra have not yet been convolved to match their respective best-fit template. The plotted spectra were extracted using the PSFdup-method described earlier as it preserved the individual spectral shapes better.

The IRTF templates used here are not listed as known members of any YMG, and are likely to be field stars and older than our target sample, therefore not necessarily reflecting the best fit to the spectral types of our sample. Nevertheless, the main difference between the IRTF models and our sample should be apparent in the line strength of gravity-sensitive features, with the IRTF templates having greater surface gravity values and thereby deeper lines. The benefit of comparing our target sample to the IRTF templates materialises from the similar resolving power of the SPeX spectrograph $(R \sim 2000)$, wavelength coverage, and high signal-to-noise ratio of the templates $(S / N \sim 100)$, as well as consistency to previous work on AstraLux binaries (e.g. Bergfors et al. 2016). The Montreal Spectral Library provides late $\mathrm{M}$ and $\mathrm{L}$ spectral types with several youth indicators such as low surface gravity, specifying subtypes with low and intermediate surface gravities, $\gamma$ and $\beta$, that our target sample can be tested against.

We complemented the initial spectral estimate by adopting a more quantitative analysis of spectral types by Rojas-Ayala et al. (2012), measuring the strength of the temperature dependent $K$-band water absorption as the $\mathrm{H}_{2} \mathrm{O}-\mathrm{K} 2$ index, calculated from the median flux in each wavelength region by

$\mathrm{H}_{2} \mathrm{O}-\mathrm{K} 2=\frac{\langle F(2.070-2.090)\rangle /\langle F(2.235-2.255)\rangle}{\langle F(2.235-2.255)\rangle /\langle F(2.360-2.380)\rangle}$.
Table 2. Empirical templates employed for spectral fitting.

\begin{tabular}{|c|c|c|}
\hline Name & Spectral type & Reference \\
\hline HD 42581 & M1V & R09 \\
\hline Gl 806 & $\mathrm{M} 2 \mathrm{~V}$ & R09 \\
\hline Gl 381 & $\mathrm{M} 2.5 \mathrm{~V}$ & R09 \\
\hline Gl 581 & $\mathrm{M} 2.5 \mathrm{~V}$ & R09 \\
\hline Gl 388 & M3V & C05, R09 \\
\hline Gl 273 & $\mathrm{M} 3.5 \mathrm{~V}$ & R09 \\
\hline Gl 213 & M4V & C05, R09 \\
\hline Gl 299 & M4V & R09 \\
\hline Gl 268AB & $\mathrm{M} 4.5 \mathrm{~V}$ & R09 \\
\hline Gl 51 & M5V & C05, R09 \\
\hline Gl 406 & M6V & C05, R09 \\
\hline Gl 644C & M7V & C05, R09 \\
\hline Gl 752B & M8V & C05, R09 \\
\hline LP $412-31$ & M8V & R09 \\
\hline 2MASS J07464256+2000321AB & L0.5 & $\mathrm{C} 05$ \\
\hline 2MASS J14392836+1929149 & L1 & $\mathrm{C} 05$ \\
\hline 2MASS J02081833+2542533 & L1 & $\mathrm{C} 05$ \\
\hline Kelu-1AB & L2 & $\mathrm{C} 05$ \\
\hline 2MASS J11463449+2230527AB & L3 & $\mathrm{C} 05$ \\
\hline 2MASS J15065441+1321060 & L3 & $\mathrm{C} 05$ \\
\hline 2MASS J00361617+1821104 & L3.5 & $\mathrm{C} 05$ \\
\hline 2MASS J22244381-0158521 & $\mathrm{L} 4.5$ & $\mathrm{C} 05$ \\
\hline 2MASS J15074769-1627386 & L5 & $\mathrm{C} 05$ \\
\hline 2MASS J03390160-2434059 & M5 $\beta$ & G15 \\
\hline 2MASS J13582164-0046262 & M5.5 $\gamma$ & G15 \\
\hline 2MASS J03350208+2342356 & M5.5 $\beta$ & G15 \\
\hline 2MASS J10284580-2830374 & M6 $\gamma$ & G15 \\
\hline 2MASS J10455263-2819303 & M6y & G15 \\
\hline 2MASS J08034469+0827000 & M6 $\beta$ & G15 \\
\hline 2MASS J20391314-1126531 & $\mathrm{M} 7 \beta$ & G15 \\
\hline 2MASS J05264316-1824315 & $\mathrm{M} 7 \beta$ & G15 \\
\hline 2MASS J03350208+2342356 & $\mathrm{M} 7.5 \beta$ & G15 \\
\hline 2MASS J08561384-1342242 & 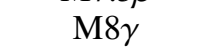 & G15 \\
\hline 2MASS J04433761+0002051 & M9 $\gamma$ & G15 \\
\hline 2MASS J11064461-3715115 & M9y & G15 \\
\hline 2MASS J09532126-1014205 & M9 $\beta$ & G15 \\
\hline 2MASS J00182834-6703130 & $\mathrm{L} 0 \gamma$ & G15 \\
\hline 2MASS J23255604-0259508 & $\mathrm{L} 1 \gamma$ & G15 \\
\hline 2MASS J05361998-1920396 & $\mathrm{L} 2 \gamma$ & G15 \\
\hline 2MASS J04185879-4507413 & $\mathrm{L} 3 \gamma$ & G15 \\
\hline 2MASS J05012406-0010452 & $\mathrm{L} 4 \gamma$ & G15 \\
\hline
\end{tabular}

References. C05 = Cushing et al. (2005), R09= Rayner et al. (2009), G15 = Gagné et al. (2015).

The equation for calculating the $\mathrm{H}_{2} \mathrm{O}-\mathrm{K} 2$ index is a modified version from Covey et al. (2010) that takes the $\mathrm{Mg} \mathrm{I}$ and Ti I atomic features that affect bright spectral measurements into account. The 1- $\sigma$ uncertainty of the $\mathrm{H}_{2} \mathrm{O}-\mathrm{K} 2$ index was calculated from a Monte Carlo simulation with random Gaussian noise based on the signal-to-noise ratio of the individual spectrum. The simulation was repeated 10000 times where the standard deviation from the measured $\mathrm{H}_{2} \mathrm{O}-\mathrm{K} 2$ index was used as the error. The resulting indices are listed in Table 3 and are used to calculate spectral types according to

$M_{\text {subtype }}=24.699-23.788\left(\mathrm{H}_{2} \mathrm{O}-\mathrm{K} 2\right)$,

where the root mean square error for the spectral subtype is \pm 0.624 . The calculated spectral types are listed in Table 3 together with the goodness-of-fit spectral types for each band. 
P. Calissendorff et al.: Characterising young M-dwarf binaries

Table 3. Near-infrared spectral types and effective temperatures for our binary pairs.

\begin{tabular}{|c|c|c|c|c|c|c|c|c|c|}
\hline Name & $\begin{array}{c}\text { Literature }^{(\dagger)} \\
\operatorname{SpT}( \pm 0.5)\end{array}$ & $\begin{array}{c}J \text {-band } \\
\text { SpT }\end{array}$ & $\begin{array}{c}H \text {-band } \\
\text { SpT }\end{array}$ & $\begin{array}{c}K \text {-band } \\
\text { SpT }\end{array}$ & $\begin{array}{c}\mathrm{H}_{2} \mathrm{O}-\mathrm{K} 2 \\
\mathrm{SpT}( \pm 0.6)\end{array}$ & $\begin{array}{l}K \text {-band } \\
T_{\text {eff }}[\mathrm{K}]\end{array}$ & $\begin{array}{c}K \text {-band } \\
\log g\end{array}$ & $\begin{array}{c}\mathrm{H}_{2} \mathrm{O}-\mathrm{K} 2 \\
T_{\text {eff }}[\mathrm{K}]\end{array}$ & $\begin{array}{c}\mathrm{H}_{2} \mathrm{O}-\mathrm{K} 2 \\
\text { index }\end{array}$ \\
\hline J0111A & M5.0 & $\mathrm{M} 7 \beta$ & M5.5 $\beta$ & M6 $\gamma$ & M3.1 & $2900_{-200}^{+200}$ & 4.0 & $3564 \pm 29$ & $0.906 \pm 0.009$ \\
\hline J0111B & M6.0 & M9 $\gamma$ & $\mathrm{L} 0 \gamma$ & $\mathrm{L} 4 \gamma$ & M9.6 & $2300_{-100}^{+200}$ & 3.5 & $2428 \pm 25$ & $0.635 \pm 0.007$ \\
\hline J0332B & M4.5 & M6r & M6 $\gamma$ & M6 $\gamma$ & M4.7 & $2800_{-200}^{+300}$ & 4.0 & $3456 \pm 30$ & $0.841 \pm 0.014$ \\
\hline J0332C & M5.5 & $\mathrm{L} 0 \gamma$ & M9 $\beta$ & $\mathrm{L} 0 \gamma$ & M5.5 & $2700_{-200}^{+300}$ & 3.5 & $3371 \pm 60$ & $0.808 \pm 0.018$ \\
\hline J0459A & M4.0 & M6r & $\mathrm{M} 2.5 \mathrm{~V}$ & M3.0V & M1.7 & $3400_{-100}^{+100}$ & 4.5 & $3734 \pm 59$ & $0.966 \pm 0.011$ \\
\hline J0459B & M5.5 & $\mathrm{M} 7 \beta$ & $\mathrm{M} 4.5 \mathrm{~V}$ & M5 $\beta$ & M4.5 & $3100_{-100}^{+200}$ & 4.5 & $3270 \pm 70$ & $0.849 \pm 0.016$ \\
\hline J0611A & M4.0 & M3V & M5V & M6 $\gamma$ & M2.9 & $3100_{-300}^{+200}$ & 4.5 & $3657 \pm 34$ & $0.916 \pm 0.011$ \\
\hline J0611B & M5.0 & M4V & M6 $\gamma$ & M6 $\gamma$ & M5.2 & $3000_{-200}^{+300}$ & 4.5 & $3411 \pm 30$ & $0.820 \pm 0.011$ \\
\hline J1014A & M4.0 & $\mathrm{M} 5 \beta$ & M5V & M4V & M2.9 & $3100_{-200}^{+100}$ & 4.5 & $3660 \pm 42$ & $0.917 \pm 0.012$ \\
\hline J1014B & M5.5 & M5V & M5.5 $\beta$ & M6 $\gamma$ & M5.2 & $2900_{-100}^{+200}$ & 4.5 & $3407 \pm 30$ & $0.818 \pm 0.011$ \\
\hline J1036B & M5.0 & M4V & M5.5 $\beta$ & M6 $\gamma$ & M5.8 & $2900_{-100}^{+100}$ & 4.5 & $3051 \pm 41$ & $0.795 \pm 0.011$ \\
\hline $\mathrm{J} 1036 \mathrm{C}$ & M5.0 & $\mathrm{M} 2.5 \mathrm{~V}$ & M5.5 $\beta$ & M6y & M4.3 & $2900_{-100}^{+200}$ & 4.5 & $3309 \pm 57$ & $0.858 \pm 0.013$ \\
\hline J2349A & M3.5 & M3V & $\mathrm{M} 2.5 \mathrm{~V}$ & $\mathrm{M} 4.5 \mathrm{~V}$ & M4.1 & $3300_{-200}^{+100}$ & 4.5 & $3513 \pm 62$ & $0.866 \pm 0.023$ \\
\hline $\mathrm{J} 2349 \mathrm{~B}$ & M4.5 & M5V & $4.5 \mathrm{~V}$ & M5.5 $\beta$ & M5.2 & $3100_{-200}^{+200}$ & 4.5 & $3412 \pm 102$ & $0.821 \pm 0.034$ \\
\hline
\end{tabular}

Notes. ${ }^{(\dagger)}$ Spectral types derived by Janson et al. (2012) based on $\left(z^{\prime}-i^{\prime}\right)$ photometry.

However, the spectral types derived from Eq. (4) may not reflect the true spectral types of the objects here, as the relation from Rojas-Ayala et al. (2012) is derived from a sample of field dwarfs, which are likely older than our target sample. We also include previous spectral type designation from the literature, where Janson et al. (2012) derived spectral types using photometry in the $z^{\prime}$ and $i^{\prime}$ bands following the procedure described in Bergfors et al. (2010), which was developed by Daemgen et al. (2007) based on the relationship between magnitudes and spectral types presented by Kraus \& Hillenbrand (2007).

\subsection{Effective temperature}

The near-infrared $K$ band is sensitive to temperature changes and is an excellent probe for spectral classification. We estimate effective temperatures, $T_{\text {eff }}$, solely from the $K$-band spectra, implementing two different approaches. Firstly, similar to our procedure for determining spectral types from the continuum shape described in Sect. 3.1, we compare our observed resolved spectra to the BT-Settl CIFIST 2011-2015 theoretical evolutionary models (Baraffe et al. 2015) by calculating the goodness-of-fit value using Eq. (1) for a grid of different temperatures and surface gravities, with steps of $\Delta T_{\text {eff }}=100 \mathrm{~K}$ and $\Delta \log g=0.5$. The theoretical spectra are prior to the fit convolved to match the resolution of the observed SINFONI spectra, and we adopt the lowest goodness-of-fit value, $G$, as our best-fit model for effective temperature, assigning those models with $G<\sqrt{2} G_{\text {best }}$ as our uncertainty. The derived effective temperatures with uncertainty ranges are shown in Table 3 . The goodness-of-fit test takes no specific weight for certain areas of the spectra, and is a direct comparison between the overall shape of the observed and template spectra. We find that within the adopted uncertainty for $G$, models typically vary in surface gravities between $\log g=3.5-5.0$, but when directly comparing only gravity sensitive lines, lower surface gravity values are favoured.

For the second approach we make use of the $\mathrm{H}_{2} \mathrm{O}-\mathrm{K} 2$ index calculated with Eq. (3), which also works as a probe for temper- ature in the $K$ band. Rojas-Ayala et al. (2012) provide the results using the $\mathrm{H}_{2} \mathrm{O}-\mathrm{K} 2$ index as a temperature indicator for the 2010 version of the BT-Settl models, finding a monotonic function, insensitive to metallicity and surface gravity changes for stars $3000 \mathrm{~K} \leq T_{\text {eff }} \leq 3800 \mathrm{~K}$ (see Table 4 in Rojas-Ayala et al. 2012). Below this boundary, however, there is a clear difference between metal-poor and metal-rich stars, and although the cause of the metallicity behaviour at the lower bound is unknown, metallicity becomes an important factor for cool stars such as those in our sample that are expected to be near the lower temperature boundary. For our purposes we assume solar metallicity for the target sample, and similar to Rojas-Ayala et al. (2012) we derive a relation between effective temperature and the $\mathrm{H}_{2} \mathrm{O}-$ K2 index for the updated BT-Settl CIFIST 2011-2015 models, calculating the $\mathrm{H}_{2} \mathrm{O}-\mathrm{K} 2$ index with Eq. (3) for each model with surface gravity of $\log g=3.5,4.0$, and 4.5 and effective temperatures in the range $T_{\text {eff }}=2000-3800 \mathrm{~K}$ (see Table 4).

\subsection{Surface gravity}

Age typically dominates the uncertainty for stellar parameters, and is one of the most difficult parameters to determine accurately. Hence, a combination of several different youth indicators is typically required in order to qualitatively determine youth; even though many individual signs may suggest a young age, they cannot by themselves establish that the star is de facto young. Some of these indicators include chromospheric and coronal activity in the form of emission features such as $\mathrm{H} \alpha$, X-ray emission, or flares, but also include signs of accretion from photometric excess or forbidden $\mathrm{O}$ I emission lines. In the optical, absorption features of the Li I $6708 \AA$ line can be used as a diagnostic for youth from lithium abundances or testing the lithium depletion boundary for low-mass stars. Another prominent method for estimating ages of stars is to adopt a "guilt by association" approach, checking for kinematic properties that are consistent with known YMGs or associations. We discuss potential YMG affiliations for individual systems in our sample in Sect. 4.1. 


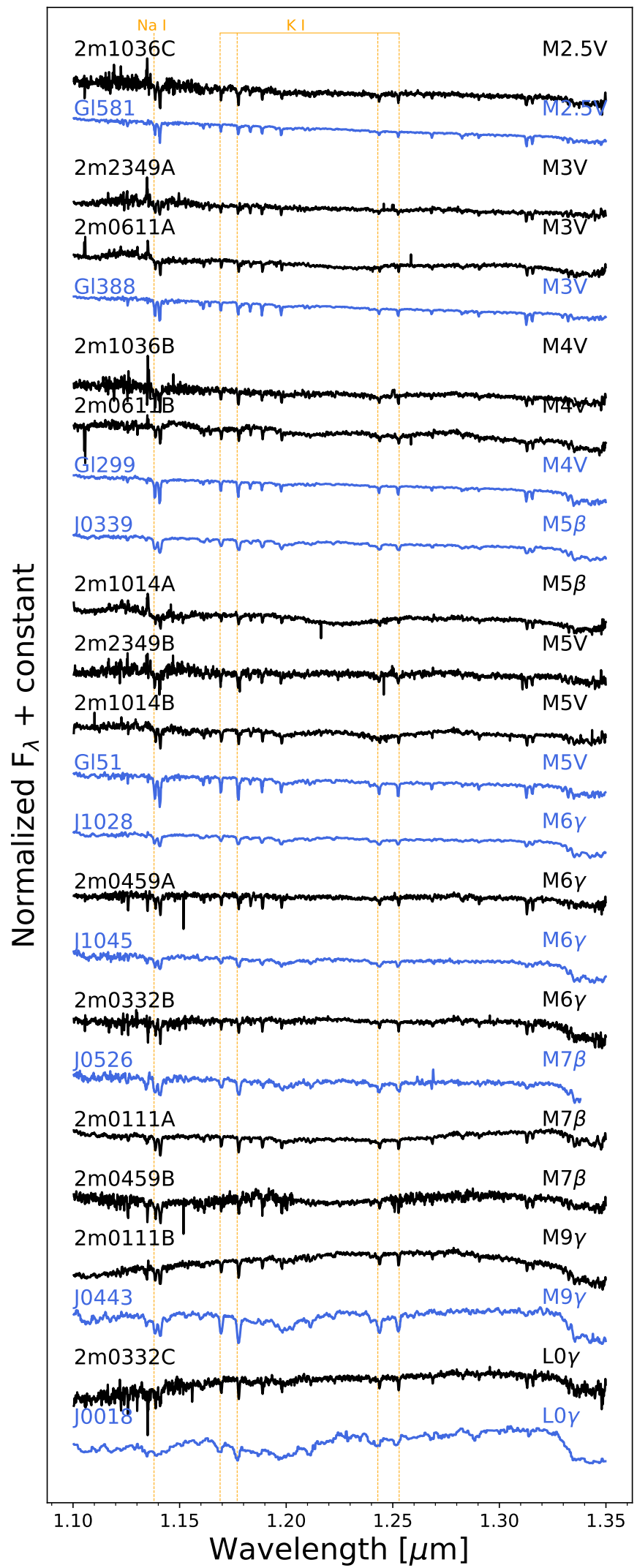

Fig. 1. $J$-band spectra of our observed binary targets in black, compared to IRTF template objects. The spectral types for our binaries are derived from the respective band continuum. Spectral features of special interest for our analysis are highlighted by the orange dashed lines.

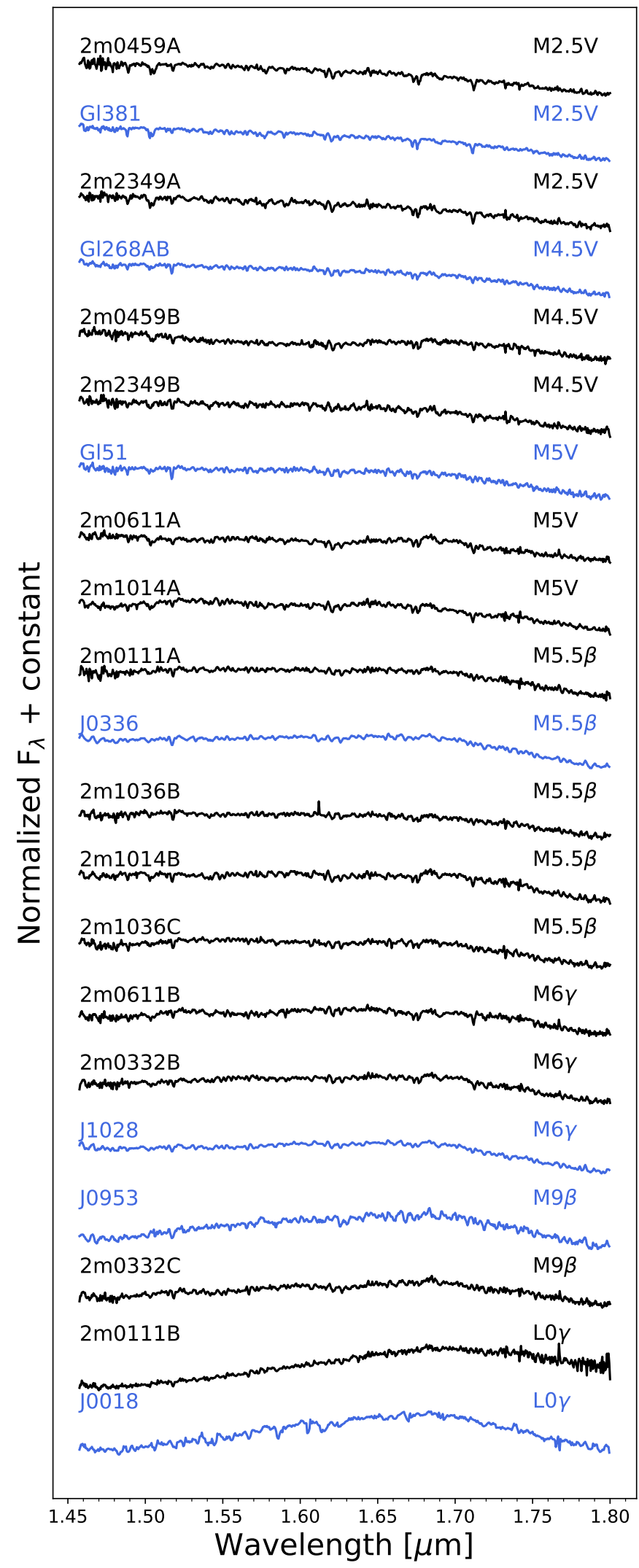

Fig. 2. Same as Fig. 1, but for the $H$ band. Some spectra display irregularities around $1.73 \mu \mathrm{m}$, which likely stems from the telluric removal procedure. 
P. Calissendorff et al.: Characterising young M-dwarf binaries

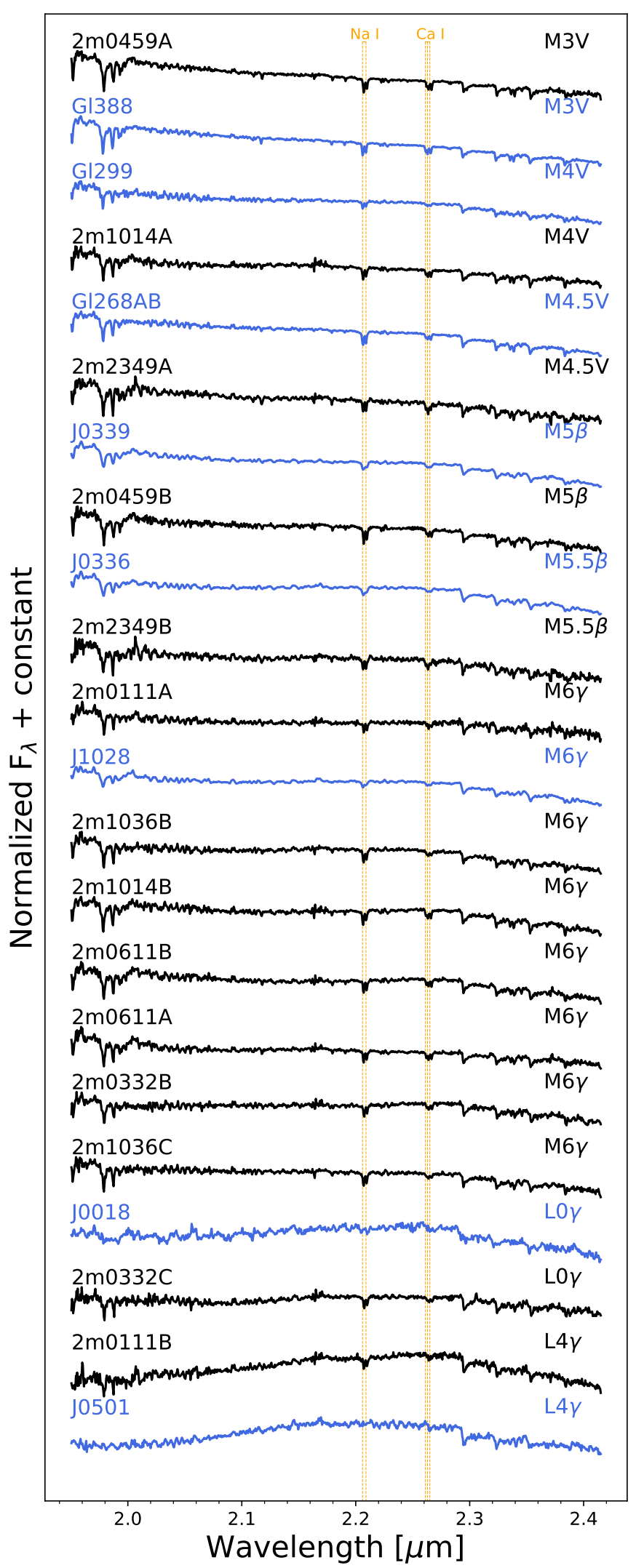

Fig. 3. Same as Fig. 1, but for the $K$ band. The bump displayed by several of our spectra at $\approx 2.16 \mu \mathrm{m}$ is likely due to a residual from the $\mathrm{Br}-\gamma$ line in the telluric standards.

Low surface gravity may also be used as a youth indicator. For instance, alkali lines in the near-infrared for a given spec-
Table 4. $\mathrm{H}_{2} \mathrm{O}-\mathrm{K} 2$ index from BT-Settl CIFIST models.

\begin{tabular}{lccc}
\hline \hline$T_{\text {eff }}[\mathrm{K}]$ & $\log g=3.5$ & $\log g=4.0$ & $\log g=4.5$ \\
\hline 2100 & 0.524 & 0.504 & 0.519 \\
2200 & 0.547 & 0.531 & 0.533 \\
2300 & 0.585 & 0.560 & 0.549 \\
2400 & 0.627 & 0.600 & 0.578 \\
2500 & 0.655 & 0.640 & 0.618 \\
2600 & 0.680 & 0.678 & 0.656 \\
2700 & 0.703 & 0.706 & 0.707 \\
2800 & 0.719 & 0.730 & 0.732 \\
2900 & 0.708 & 0.741 & 0.755 \\
3000 & 0.714 & 0.754 & 0.780 \\
3100 & 0.741 & 0.786 & 0.810 \\
3200 & 0.762 & 0.801 & 0.833 \\
3300 & 0.789 & 0.829 & 0.856 \\
3400 & 0.815 & 0.860 & 0.879 \\
3500 & 0.861 & 0.889 & 0.909 \\
3600 & 0.895 & 0.916 & 0.942 \\
3700 & 0.931 & 0.988 & 0.960 \\
3800 & 0.946 & 1.006 & 0.977 \\
\hline
\end{tabular}

tral subtype are sensitive to gravity changes and will display a reduced strength compared to main sequence stars (see e.g. Gorlova et al. 2003; Kirkpatrick et al. 2006; Allers et al. 2007). Mentuch et al. (2008) surveyed lithium depletion for young stellar associations, including the 20-30 Myr Tucana-Horologium and $\beta$ pic groups, of which several of our target objects are highprobability members. Using gravity sensitive spectral features, they report surface gravities for most low-mass members to be around $\log g=4.0-4.8$, which is what we can expect from our target sample. Furthermore, David et al. (2019) reports surface gravities of $\log g \approx 4.1$ for very young ( $\leq 10 \mathrm{Myr}$ ) low-mass binaries in Upper Scorpius, and we can expect our target sample to have near but higher surface gravity values in comparison to Upper Scorpius objects of similar mass.

In this paper we check our observed spectra for low surface gravity by measuring EWs of the gravity sensitive $\mathrm{NaI}$ doublet at $1.138 \mu \mathrm{m}$ and the $\mathrm{KI}$ lines at $1.169,1.177,1.243$, and $1.253 \mu \mathrm{m}$ in the $J$ band. The procedure is similar to what is described in Bonnefoy et al. (2014) and Bergfors et al. (2016), following the prescription of Sembach \& Savage (1992). Since M-dwarf spectra are greatly affected by molecular and broad-band features in the continuum, we calculate a pseudocontinuum using adjacent regions to the features of interest which are free of other atomic features. The EWs are calculated according to a Riemann sum expression of the integrated area over the wavelength region of interest as

$\mathrm{EW}_{\lambda} \simeq \sum_{i=0}^{n}\left[1-\frac{F\left(\lambda_{i}\right)}{F_{\mathrm{c}}\left(\lambda_{i}\right)}\right] \Delta \lambda_{i}$

where $F\left(\lambda_{i}\right)$ and $F_{\mathrm{c}}\left(\lambda_{i}\right)$ are the respective line flux and pseudocontinuum flux of the wavelength interval $\Delta \lambda_{i}$.

The uncertainties for the EW measurements are obtained via a Monte Carlo approach where a Gaussian noise based on the signal-to-noise ratio of the spectra is added to the observed spectra. The EW of each feature is then calculated using Eq. (5) and the procedure is repeated 1000 times, where the standard deviation from the EW of the observed spectra is adopted as the uncertainty. The EWs and their uncertainties are listed in Table 5. 
Table 5. Equivalent widths of gravity sensitive features in $J$ band.

\begin{tabular}{|c|c|c|c|c|c|}
\hline Name & $\begin{array}{c}\mathrm{Na} \mathrm{I}-1.138 \mu \mathrm{m} \\
{[\AA]}\end{array}$ & $\begin{array}{c}\mathrm{K} \mathrm{I}-1.169 \mu \mathrm{m} \\
{[\AA]}\end{array}$ & $\begin{array}{c}\mathrm{K} \mathrm{I}-1.177 \mu \mathrm{m} \\
{[\AA]}\end{array}$ & $\begin{array}{c}\mathrm{K} \mathrm{I}-1.243 \mu \mathrm{m} \\
{[\AA]}\end{array}$ & $\begin{array}{c}\mathrm{KI}-1.253 \mu \mathrm{m} \\
{[\AA]}\end{array}$ \\
\hline J0111A & $4.10 \pm 0.17$ & $1.13 \pm 0.13$ & $2.54 \pm 0.09$ & $1.03 \pm 0.10$ & $1.37 \pm 0.11$ \\
\hline J0111B & $5.46 \pm 0.41$ & $1.53 \pm 0.19$ & $2.21 \pm 0.11$ & $1.46 \pm 0.13$ & $1.16 \pm 0.11$ \\
\hline J0332B & $2.48 \pm 0.45$ & $0.46 \pm 0.17$ & $1.54 \pm 0.19$ & $1.00 \pm 0.10$ & $0.97 \pm 0.11$ \\
\hline J0332C & $2.56 \pm 1.01$ & $1.87 \pm 0.35$ & $3.03 \pm 0.20$ & $0.97 \pm 0.17$ & $1.72 \pm 0.15$ \\
\hline J0459A & $3.00 \pm 0.38$ & $1.22 \pm 0.18$ & $2.44 \pm 0.12$ & $1.05 \pm 0.09$ & $0.22 \pm 013$ \\
\hline J0459B & $4.23 \pm 0.47$ & $0.31 \pm 0.40$ & $0.07 \pm 0.38$ & $0.89 \pm 0.19$ & $2.27 \pm 0.30$ \\
\hline J0611A & $4.30 \pm 0.50$ & $0.88 \pm 0.17$ & $1.38 \pm 0.14$ & $1.07 \pm 0.20$ & $1.09 \pm 0.20$ \\
\hline J0611B & $3.44 \pm 0.26$ & $1.17 \pm 0.22$ & $1.81 \pm 0.20$ & $0.37 \pm 0.11$ & $0.51 \pm 0.21$ \\
\hline J1014A & $3.48 \pm 0.60$ & $0.66 \pm 0.17$ & $1.77 \pm 0.12$ & $1.54 \pm 0.19$ & $0.39 \pm 0.19$ \\
\hline J1014B & $3.33 \pm 0.37$ & $1.66 \pm 0.22$ & $2.33 \pm 0.06$ & $1.19 \pm 0.18$ & $1.24 \pm 0.27$ \\
\hline J1036B & $7.31 \pm 0.83$ & $1.47 \pm 0.29$ & $3.38 \pm 0.28$ & $1.51 \pm 0.18$ & $2.88 \pm 0.23$ \\
\hline $\mathrm{J} 1036 \mathrm{C}$ & $6.02 \pm 0.72$ & $1.22 \pm 0.31$ & $3.03 \pm 0.26$ & $1.88 \pm 0.19$ & $0.32 \pm 0.21$ \\
\hline J2349A & $3.74 \pm 0.57$ & $0.83 \pm 0.22$ & $2.42 \pm 0.14$ & $0.90 \pm 0.17$ & $2.41 \pm 0.19$ \\
\hline $\mathrm{J} 2349 \mathrm{~B}$ & $5.46 \pm 0.53$ & $0.67 \pm 0.32$ & $1.61 \pm 0.26$ & $-0.55 \pm 0.41$ & $3.34 \pm 0.35$ \\
\hline
\end{tabular}

Equivalent widths are calculated using Eq. (5), where pseudo-continuum regions are fitted using Legendre polynomials of third order locally around the line of interest, which are then interpolated to the feature wavelength. The reference wavelengths used for the line feature and pseudo-continuum regions are shown in Table 6, where the pseudo-continuum regions are calculated using wavelength regions between $\lambda_{1}-\lambda_{2}$ and $\lambda_{3}-\lambda_{4}$, and the line feature from the region between $\lambda_{2}$ and $\lambda_{3}$. We list the measured EWs in Table 5, which are also plotted against spectral type in Fig. 4 together with EWs for IRTF SpeX library of field dwarfs and a sample of young stars <10 Myr from Manara et al. (2013) for comparison. We find that objects in our sample with estimated spectral types of $\sim$ M5 or later have systematically lower line strength, and thereby also lower surface gravity, compared to the older field dwarf IRTF sample. Conversely, our sample has greater line strength than the very young stars from Manara et al. (2013), which is consistent with the estimated ages for our target sample, being part of YMGs and having ages intermediate to those of the old field and the very young populations. However, given the difficulties with the deblending procedure described in Sect. 2.2, it is possible that the lines are diluted by residual companion contamination and the uncertainties are underestimated.

We further test the validity of the derived surface gravities for our objects by comparing the observed spectra to synthetic spectra from the BT-Settl CIFIST 2011-2015 theoretical models, varying the surface gravities of the models at fixed metallicity and for specific temperatures. Best-fit calculations are performed using Eq. (1) for constrained wavelength areas around the gravity sensitive features in the $J$ band shown in Table 6 . The synthetic spectra indicate that lower temperature objects $(\lesssim 2800 \mathrm{~K})$ are more sensitive to changes in surface gravity, and we can test whether the observed SINFONI spectra are consistent with being young objects. At higher temperatures the sensitivity is more modest and the best-fit procedure develops into a degenerate issue. A discussion from the results of this test is presented Sect. 4 for each individual system.

\subsection{Astrometry and photometry}

From the spectral extraction procedure described in Sect. 2.2 we also obtained astrometric positions and resolved photometry for our binary components. To calibrate the photometric measure-
Table 6. Reference wavelengths for Na I and K I EWs.

\begin{tabular}{lcccc}
\hline \hline Feature & $\begin{array}{c}\lambda_{1} \\
{[\mu \mathrm{m}]}\end{array}$ & $\begin{array}{c}\lambda_{2} \\
{[\mu \mathrm{m}]}\end{array}$ & $\begin{array}{c}\lambda_{3} \\
{[\mu \mathrm{m}]}\end{array}$ & $\begin{array}{c}\lambda_{4} \\
{[\mu \mathrm{m}]}\end{array}$ \\
\hline $\mathrm{Na} \mathrm{I}-1.138 \mu \mathrm{m}$ & 1.1270 & 1.1360 & 1.1420 & 1.1580 \\
$\mathrm{~K} \mathrm{I}-1.169 \mu \mathrm{m}$ & 1.1560 & 1.1670 & 1.1710 & 1.1760 \\
$\mathrm{~K} \mathrm{I}-1.177 \mu \mathrm{m}$ & 1.1710 & 1.1750 & 1.805 & 1.1820 \\
$\mathrm{~K} \mathrm{I}-1.243 \mu \mathrm{m}$ & 1.2390 & 1.2415 & 1.2455 & 1.2490 \\
$\mathrm{~K} \mathrm{I}-1.253 \mu \mathrm{m}$ & 1.2455 & 1.2500 & 1.2550 & 1.2670 \\
\hline
\end{tabular}

ments of our targets we compare the unresolved 2MASS magnitudes (Skrutskie et al. 2006) of our targets to magnitudes of the standard star observed together with the respective target. The magnitudes are sequentially scaled to the brightness difference of the components to obtain resolved photometry. Since the photometry is performed in a subset of wavelengths not entirely covering the full width of the 2MASS photometric measurements, albeit based on unresolved 2MASS data, we consider our astrometric and photometric measurements to be in passbands of $J^{\prime}\left(1.10 \leq \lambda_{J^{\prime}} \leq 1.35 \mu \mathrm{m}\right), H^{\prime}\left(1.45 \leq \lambda_{H^{\prime}} \leq 1.80 \mu \mathrm{m}\right)$, and $K^{\prime}\left(1.945 \leq \lambda_{K^{\prime}} \leq 2.415 \mu \mathrm{m}\right)$. The difference in magnitudes for each binary pair is listed in Table 7 , where the error is quadratically added from the 2MASS magnitude errors and the photon noise from the spectral extraction. Estimated magnitudes for all resolved components in the triple systems J0332 and J1036 are shown in Table 8.

We measure astrometric positions by collapsing the datacubes in the specific band of interest, and perform the same positional grid search as we did for individual wavelength slices for the spectral extraction with the PSFdup reference. The reason for collapsing the datacube in this context is to increase the signal-to-noise ratio for more precise positions. We do this for each band individually, where the mean of the separation and positional angle for all three bands are shown in Table 7, with the standard deviation from the mean as the uncertainty.

\section{Results and discussion}

\subsection{Individual systems}

Bergfors et al. (2010) estimated that the AstraLux sample consists of stars younger than $1 \mathrm{Gyr}$, with most being even younger 
than $\approx 600$ Myr based on their selection from the catalogue of stars with strong coronal emission and low tangential velocity by Riaz et al. (2006). More recently, large surveys of space velocities and kinematics have revealed that some of these AstraLux targets are high-probability members of YMGs and associations (e.g. Malo et al. 2013; Gagné et al. 2014a,b). Below we provide for each individual system in our sample a discussion on the findings in our survey as well as additional youth indicators such as YMG membership.

2MASS J01112542+1526214. The system has the largest separation of the binary pairs in our sample, over $1^{\prime \prime}$. We find from the spectral type and spectral EW analysis that the young age is consistent when compared to both the older field population and to the very young sample. J0111B is the coolest object in our survey, and has an observed spectrum that our best-fit calculations find to be consistent with an L-type template. When we test synthetic spectra for spectral line feature changes with respect to change in surface gravity, we find a best fit for low surface gravity, $\log g=3.5$, for a low temperature of $T_{\text {eff }}=2300 \mathrm{~K}$ for the binary component J0111B. We further find that although the strength of the spectral line features is compatible for higher temperature models, the continuum fit is only consistent with low-temperature models, which supports the notion of the object being young, and explains the difference in line strength compared to the IRTF models seen in Fig. 1.

2MASS J03323578+2843554. A triple system where the outer binary BC components are likely of later spectral types, $\sim$ M6 and L0, according to both our continuum and spectral feature analysis. Components B and C in the outer binary of J0332 are both compatible with low surface gravity, and our best-fit test yields $\log g=3.5$ for the pair, which also has relatively low effective temperatures of $T_{\text {eff }}=2800 \mathrm{~K}$ and $2700 \mathrm{~K}$, respectively, consistent with a young age for the system. We find from the $\mathrm{H}_{2} \mathrm{O}-\mathrm{K} 2$ index a discrepancy in the effective temperature measurement, obtaining higher values of $\approx 3400 \mathrm{~K}$. However, for these higher temperatures the continuum of the observed spectra deviates heavily from the synthetic models, and is more consistent with the lower temperature models with respect to both continuum and spectral line features.

2MASS J04595855-0333123. The system consists of one brighter $\sim \mathrm{M} 2.5$ primary with a $\sim \mathrm{M} 4.5$ secondary component. The $J$-band spectrum fit for the system shows a discrepancy compared to the other spectral type estimates. We find no obvious reason as to why we obtain this discrepancy, but we note that we obtain the worst fit for each component in this band, and that perhaps a suitable template, for example a young and early $\mathrm{M}$ type, was lacking. Our best fit when comparing to theoretical model spectra suggest that the primary and secondary components have effective temperatures of $T_{\text {eff }}=3400$ and $3100 \mathrm{~K}$, respectively. We obtain a best fit for models with surface gravity of $\log g=4.5$, but at this temperature range lower surface gravities are also consistent with the shape of the spectra, and the gravity sensitive line features in the $J$ band are more consistent with a lower surface gravity of $\log g \approx 4.0$.

2MASS J06112997-7213388. We find for the continuum fit spectral types of M3-6 and M4-6 for the primary and secondary, respectively, suggesting that our continuum fit was poorly constrained for this system. The fitted models within the adopted uncertainty of $\sqrt{2}$ of the lowest goodness-of-fit value range a mix of older IRTF and younger subspectral types, suggesting that early $\mathbf{M}$ types younger than the templates probed here $(\leq \mathrm{M} 5 \gamma / \beta)$ could potentially be a better match for the

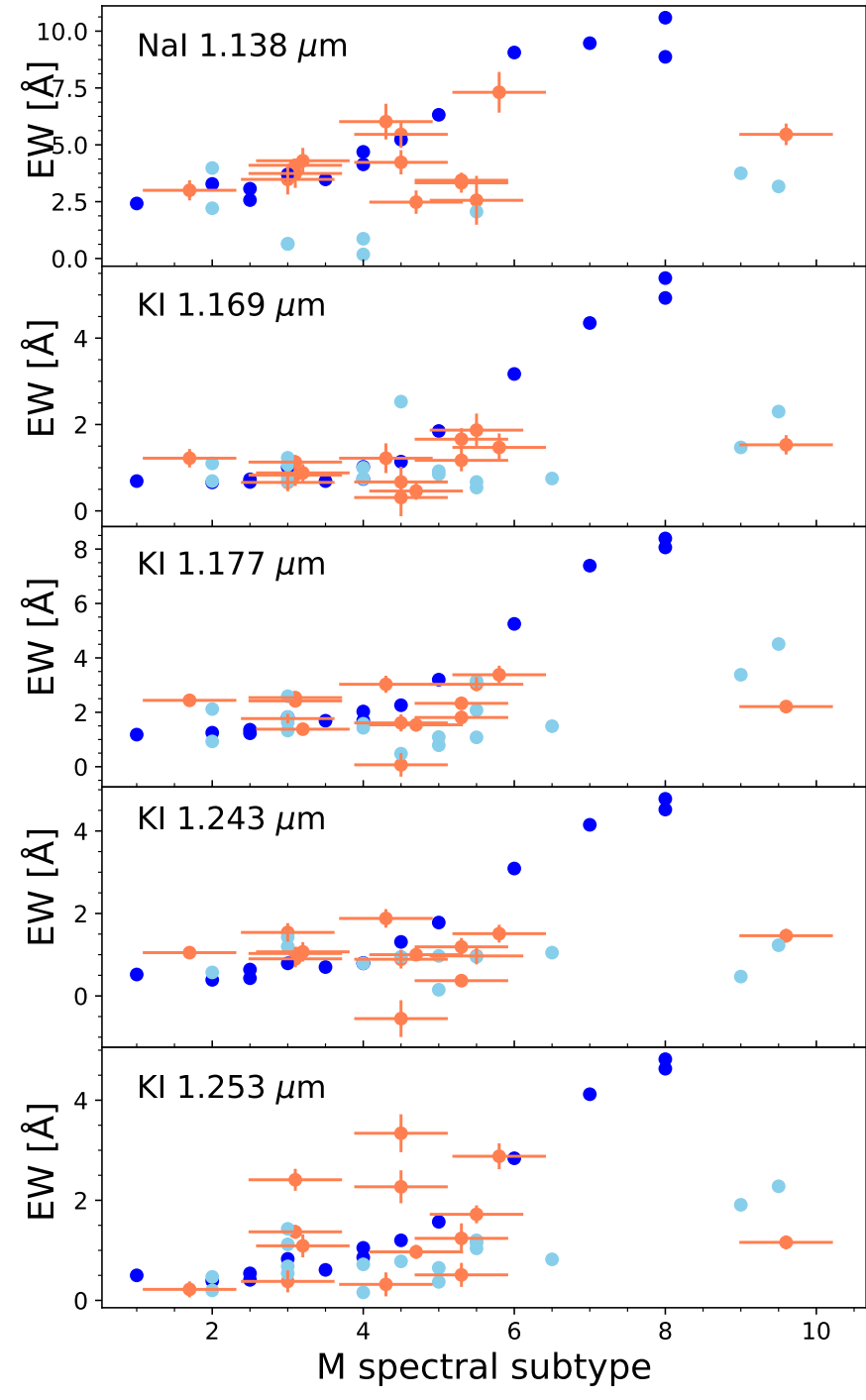

Fig. 4. Equivalent widths of surface gravity sensitive features, listed in Table 5. Our measured EWs from SINFONI spectra (orange circles) are compared to measured EWs from older field stars in the IRTF sample (dark blue circles) and young < 10 Myr stars from Manara et al. (2013) (light blue circles). The spectral types for our target sample are derived from the $\mathrm{H}_{2} \mathrm{O}-\mathrm{K} 2$ index in Table 3.

system. The $\mathrm{H}_{2} \mathrm{O}-\mathrm{K} 2$ index analysis, on the other hand, suggests that the primary is of M2.9 \pm 0.6 spectral type, and the secondary M5. $2 \pm 0.6$. We find from our best-fit theoretical model that the components have effective temperatures of about $3100 \mathrm{~K}$ and $3000 \mathrm{~K}$, respectively, with the overall best goodness-of-fit for surface gravity of $\log g=4.5$. The $\mathrm{H}_{2} \mathrm{O}-\mathrm{K} 2$ index calculations suggest higher temperatures, however, with effective temperatures of $T_{\mathrm{eff}} \approx 3600$ and 3400 for the individual components. The $J$-band individual spectral features show a better fit for slightly lower surface gravities $(\log g \approx 4.0-4.5)$ consistent with the system being of young age.

2MASSJ10140807-7636327. A well-separated binary with components of similar brightness. We obtain individual spectral types of $\approx$ M5 and $\approx$ M5.5 for the primary and secondary, respectively. We find the best-fit effective temperatures for the components to be $T_{\text {eff }}=3100$ and $2900 \mathrm{~K}$, respectively, with a best-fit surface gravity of $\log g=4.5$; however, the $J$-band spectral feature analysis suggests lower surface gravities, and we obtain better fits for $\log g \lesssim 4.0$. However, we find better 
Table 7. Astrometric and photometric measurements for binary pairs.

\begin{tabular}{lccccc}
\hline \hline Name & $\begin{array}{c}\text { Sep } \\
{[\mathrm{mas}]}\end{array}$ & $\begin{array}{c}\mathrm{PA}^{(\dagger)} \\
{\left[{ }^{\circ}\right]}\end{array}$ & $\begin{array}{c}\Delta J^{\prime} \\
{[\mathrm{mag}]}\end{array}$ & $\begin{array}{c}\Delta H^{\prime} \\
{[\mathrm{mag}]}\end{array}$ & $\begin{array}{c}\Delta K^{\prime} \\
{[\mathrm{mag}]}\end{array}$ \\
\hline J0111AB & $1040.9 \pm 13$ & $9.5 \pm 1.4$ & $0.98 \pm 0.03$ & $1.30 \pm 0.05$ & $0.63 \pm 0.05$ \\
$\mathrm{~J} 0332 \mathrm{BC}$ & $86.3 \pm 2.4$ & $19.6 \pm 1.4$ & $0.87 \pm 0.02$ & $0.74 \pm 0.02$ & $0.65 \pm 0.02$ \\
J0459AB & $142.6 \pm 2.0$ & $53.8 \pm 1.2$ & $1.19 \pm 0.02$ & $1.50 \pm 0.03$ & $1.21 \pm 0.03$ \\
J0611AB & $177.6 \pm 3.2$ & $30.2 \pm 0.4$ & $0.37 \pm 0.03$ & $0.31 \pm 0.03$ & $0.26 \pm 0.03$ \\
$\mathrm{~J} 1014 \mathrm{AB}$ & $283.9 \pm 0.1$ & $7.4 \pm 0.1$ & $-0.05 \pm 0.03$ & $0.05 \pm 0.03$ & $-0.02 \pm 0.02$ \\
J1036BC & $99.8 \pm 2.9$ & $8.6 \pm 1.7$ & $0.02 \pm 0.02$ & $0.04 \pm 0.03$ & $-0.00 \pm 0.03$ \\
J2349AB & $208.1 \pm 1.1$ & $37.2 \pm 0.1$ & $1.13 \pm 0.03$ & $1.07 \pm 0.03$ & $1.07 \pm 0.02$ \\
\hline
\end{tabular}

Notes. ${ }^{(\dagger)}$ Positional Angles have not been corrected for True North.

goodness-of-fit values for the individual spectral features in the $J$ band when applying synthetic models with higher $T_{\text {eff }}$ values compared our $K$-band analysis; the temperatures are also more consistent with the values derived from the $\mathrm{H}_{2} \mathrm{O}-\mathrm{K} 2$ index of $T_{\text {eff }} \approx 3600$ and 3400, respectively.

2MASSJ10364483+1521394. We estimate the spectral types for the outer binary to be between M2.5 and 6.0, with a favour towards later types from the continuum fit. Only the $J$-band spectra yield good fits for early M types; the observations were of quite poor quality with low signal-to-noise ratio for the outer binary pair. We redo our spectral template fit for the outer binary of J1036 when it is unresolved, finding a best fit for a M4V type. This may suggest that the signal-to-noise ratio in the $J$ band was too low for our astrometric procedure and deblending process to provide accurate brightness scalings of the two components in the outer binary. The $J$-band spectrum was corrected from the telluric contribution using a different standard star compared to the $H+K$ band, and the only system in our sample to use different standard telluric stars for different bands. Calissendorff et al. (2017) placed orbital constraints for the outer binary and estimated individual dynamical masses to be $\approx 0.24 M_{\odot}$ for each component. The theoretical model best-fit procedure suggests an effective temperature of $T_{\text {eff }}=2900 \mathrm{~K}$ for each component, and a surface gravity of $\log g=4.5$ that is consistent with the individual spectral line analysis. The EW analysis also show the strongest lines for this system, further supporting an older age estimate compared to the rest of our sample.

2MASS J23495365+2427493. We find from our continuum spectral fit that the primary is of $\mathrm{M} 3.5 \pm 1.0$ spectral type and the secondary has spectral type M5.0 \pm 0.5 , which are somewhat earlier than the estimates we obtain from the $\mathrm{H}_{2} \mathrm{O}-\mathrm{K} 2$ index analysis for each component. We estimate the effective temperature of the components as $T_{\text {eff }}=3300$ and $3000 \mathrm{~K}$, respectively, with surface gravity of $\log g=4.5$. Similar surface gravities are found for the individual spectral line features, with a better fit tendency towards higher surface gravities $\approx 4.5$. The empirical spectral template fit and synthetic theoretical models both seem to suggest that the system could be somewhat older than the rest of our sample, and belong to the field rather than any known YMG. Nevertheless, the observations of 2M2349 have the highest uncertainty due to only one out of five frames being used in our analysis.

\subsection{Hertzsprung-Russel diagram}

Theoretical evolutionary models can also provide additional information on the age and mass of a system when compared
Table 8. Resolved photometry for triple systems.

\begin{tabular}{lccc}
\hline \hline Component & $\begin{array}{c}J^{\prime} \\
{[\mathrm{mag}]}\end{array}$ & $\begin{array}{c}H^{\prime} \\
{[\mathrm{mag}]}\end{array}$ & $\begin{array}{c}K^{\prime} \\
{[\mathrm{mag}]}\end{array}$ \\
\hline J0332A & 9.83 & 9.45 & 9.16 \\
J0332B & 10.66 & 10.02 & 9.77 \\
J0332C & 11.53 & 10.76 & 10.42 \\
J1036A & 9.18 & 8.90 & 8.57 \\
J1036B & 10.71 & 9.70 & 9.48 \\
J1036C & 10.73 & 9.74 & 9.48 \\
\hline
\end{tabular}

Notes. Magnitudes are scaled from their relative brightness and unresolved 2MASS photometry. Errors are of the order of \pm 0.02 mag.

to dynamical masses. One of the binaries in our sample has its dynamical mass constrained from an orbital fit already, J1036 (Calissendorff et al. 2017), with each binary component having an individual mass of $0.24 \pm 0.07 M_{\odot}$, which we can use for direct comparison to the measured luminosities, temperatures, and masses in a Hertzsprung-Russel diagram (HRD). We compare the $K$-band magnitudes and effective temperatures of the individual binary components for J1036 to six different isochrones of ages 10, 20, 30, 50, 120, and $400 \mathrm{Myr}$ from the BHAC15 models (Baraffe et al. 2015) shown in Fig. 5. The isochrone ages are chosen to better represent the different YMG ages associated with the systems of interest, and that after $\sim 400 \mathrm{Myr}$ the objects enter the main sequence track, and the models do not change much at these low stellar masses. Several mass-tracks are displayed in the figure, highlighting theoretical masses at $0.5,0.4,0.3,0.2,0.1,0.08$ and $0.06 M_{\odot}$. Calissendorff et al. (2017) found a discrepancy of $\approx 30 \%$ between the dynamical mass-estimate and the theoretical masses from the evolutionary models, with the models under-predicting the mass. We find similar results for the B component when we compare the $K$-band magnitudes to measured effective temperature from the $\mathrm{H}_{2} \mathrm{O}-\mathrm{K} 2$ index measurements, that the models suggest much lower mass compared to the dynamical mass estimate. We also find from the isochrone fit a younger age for the system than anticipated. However, for the $\mathrm{C}$ component the isochronal fit is consistent with the dynamical mass estimate. It is unlikely that the temperature is so different for the two components in the J1036 system as they are of equal mass and brightness. We attribute the observed discrepancy in our $\mathrm{H}_{2} \mathrm{O}-\mathrm{K} 2$ index measurements to issues with the astrometric calculations and brightness scaling for the B component, as its halo is not entirely within the field of view, and that the uncertainty is underestimated. 

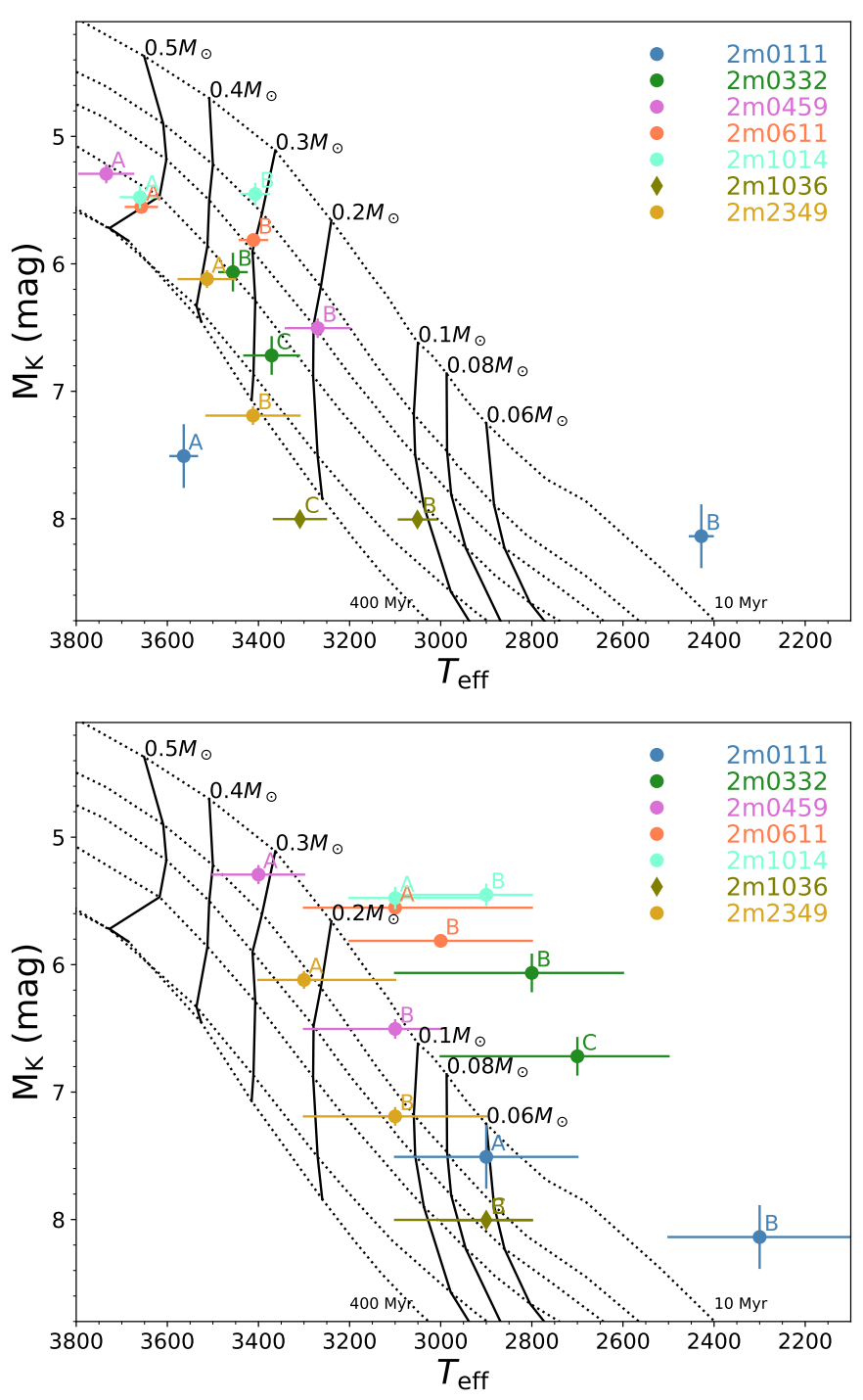

Fig. 5. Isochrones from the BHAC (Baraffe et al. 2015) evolutionary models plotted as 2MASS $K$-band magnitudes as a function of effective temperature. The isochrones span ages 10, 20, 30, 50, 120, and $400 \mathrm{Myr}$ (from top to bottom). Mass tracks at 0.06, 0.08, 0.1, 0.2, 0.3, 0.4, and $0.5 M_{\odot}$ are displayed going across the isochrones. The binary pairs are included in the figure as coloured circles where the absolute magnitude is calculated from the resolved $K^{\prime}$-band magnitudes in Table 7 with either Gaia DR2 parallaxes or statistical distances from Malo et al. (2013). Top panel: effective temperatures (see Table 3) derived from the $\mathrm{H}_{2} \mathrm{O}-\mathrm{K} 2$ indices; Bottom panel: effective temperature estimated from the $K$-band continuum fitted to theoretical models. Effective temperatures are listed in Table 3. The bottom panel indicates younger ages, and that the uncertainty for the effective temperature derived from the $\mathrm{H}_{2} \mathrm{O}-\mathrm{K} 2$ index is underestimated. The J1036 system is depicted by diamonds in the plots, and is the only binary pair for which a dynamical mass estimate exists, estimated to be $0.24 \pm 0.07 M_{\odot}$ for each individual component (Calissendorff et al. 2017).

Nevertheless, when comparing the effective temperature of $2800-3100 \mathrm{~K}$ obtained from the $K$-band continuum spectral fit instead, we find the components to be more consistent with each other, and within $2 \sigma$ of the dynamical mass estimate and age range of the Ursa Majoris moving group or field population. The spectral EW feature analysis indicates that the system has lower surface gravity compared to the older field population, but also one of the highest compared to the other binaries in our sam- ple, which is consistent with the age estimate of young field or $\approx 400$ Myr Ursa Majoris moving group.

The absolute magnitude in $K$ band is calculated here using the Gaia DR2 parallax for the J1036 system, corresponding to a distance of $d=19.75 \pm 0.06 \mathrm{pc}$, and slightly less than the distance used in Calissendorff et al. (2017), even though it has a negligible impact on the absolute magnitude $(\leq 0.04 \Delta \mathrm{mag})$ and isochrone fit.

We also include the other binary pairs in the HRD in Fig. 5, but without dynamical mass-estimates the results become more difficult to gauge. Nevertheless, we find most objects to be consistent with young ages from the isochrone analysis, but that most of them have underpredicted masses when we apply the effective temperatures derived from the $\mathrm{H}_{2} \mathrm{O}-\mathrm{K} 2$ index.

\section{Summary and conclusions}

We provide a near-infrared spectral analysis of seven close visual M-dwarf binaries from the AstraLux multiplicity survey, constraining spectral types for all resolved components in our study. Although the binary components are spatially resolved with the help of adaptive optics, PSF overlaps typically persist. We use PSF matching techniques to minimise cross-contamination of the spectra resulting from this overlap. Nonetheless, imperfections in the PSF matching can still potentially cause some residual contamination in the closest or highest-contrast binaries. We further probe the targets for low surface gravity by scrutinising EW measurements of gravity sensitive alkali lines and other signs of youth, finding them all to have some or several indicators that they are of young age. The validation of young ages for these systems will be particularly beneficial for dynamical mass studies in the future as these systems may be used as exemplary benchmarks for calibrating theoretical evolutionary models.

Kinematics including proper motions and trigonometric parallax measurements from Gaia DR2 are missing for the J0111, J0459, and J1014 binaries. Regardless, kinematic measurements from the ground and spectroscopic distance measurements place these systems as members of YMGs with a high level of probability (Malo et al. 2013). Our EW analysis is consistent with this prediction that they are indeed young, but future Gaia data releases may provide firmer constraints on not only the kinematics of these systems but also YMGs and associations as a whole.

The main purpose of this study was to characterise symbolic binaries that can be used as benchmarks to test against theoretical models, which tend to systematically underpredict masses for low-mass stars $M \leq 0.5 M_{\odot}$ (Hillenbrand \& White 2004; Calissendorff et al. 2017; Rodet et al. 2018). We find our sample to be consistent with young ages, in the range 20-400 Myr according to the YMG membership probabilities obtained from the BANYAN $\Sigma$-tool. Compared to the other AstraLux sample of seven low-mass M-dwarf binaries from Bergfors et al. (2016), our sample is younger overall. For the Bergfors et al. (2016) sample we find with the BANYAN $\Sigma$ tool that only the J06134539-2352077 binary system belongs to the Argus moving group, while the rest are either part of the field or have insufficient data for a rigorous assessment of Bayesian YMG membership probabilities. Thus, the systems studied in our sample will become part of a larger sample of well-studied young low-mass M-dwarf binaries, which together with the Bergfors et al. (2016) extends 14 characterised binaries over a wide range of ages that can be tested against evolutionary models once orbital constraints can be placed. As 
the AstraLux is an ongoing astrometric monitoring survey, and since these systems have predicted orbital periods of just a few years to a few decades, we can expect to soon be able to constrain the dynamical masses for some of these emblematic binaries.

Acknowledgements. We thank the referee, Bárbara Rojas-Ayala, for the insightful comments and advice that helped improve the manuscript. M.J. gratefully acknowledges funding from the Knut and Alice Wallenberg foundation. This publication makes use of data products from the Two Micron All Sky Survey, which is a joint project of the University of Massachusetts and the Infrared Processing and Analysis Center/California Institute of Technology, funded by the National Aeronautics and Space Administration and the National Science Foundation. This research has benefitted from the Montreal Brown Dwarf and Exoplanet Spectral Library, maintained by Jonathan Gagné. This work has made use of data from the European Space Agency (ESA) mission Gaia (https: //www . cosmos.esa.int/gaia), processed by the Gaia Data Processing and Analysis Consortium (DPAC, https://www. cosmos.esa.int/web/gaia/dpac/ consortium). Funding for the DPAC has been provided by national institutions, in particular the institutions participating in the Gaia Multilateral Agreement.

\section{References}

Allers, K. N., Jaffe, D. T., Luhman, K. L., et al. 2007, ApJ, 657, 511 Baraffe, I., Homeier, D., Allard, F., \& Chabrier, G. 2015, A\&A, 577, A42

Bergfors, C., Brandner, W., Janson, M., et al. 2010, A\&A, 520, A54

Bergfors, C., Brandner, W., Bonnefoy, M., et al. 2016, MNRAS, 456, 2576

Bonnefoy, M., Chauvin, G., Dumas, C., et al. 2009, A\&A, 506, 799

Bonnefoy, M., Chauvin, G., Lagrange, A.-M., et al. 2014, A\&A, 562, A127

Bonnet, H., Strobele, S., Biancat-Marchet, F., et al. 2003, in Adaptive Optical System Technologies II, Proc. SPIE, 4839

Bouy, H., Martín, E. L., Brandner, W., et al. 2008, A\&A, 481, 757

Bowler, B. P., Shkolnik, E. L., Liu, M. C., et al. 2015, ApJ, 806, 62

Burgasser, A. J., Reid, I. N., Siegler, N., et al. 2007, Protostars and Planets V, eds. B. Reipurth, D. Jewitt, \& K. Keil (Tucson: University of Arizona Press), 427

Calissendorff, P., \& Janson, M. 2018, A\&A, 615, A149

Calissendorff, P., Janson, M., Köhler, R., et al. 2017, A\&A, 604, A82

Calissendorff, P., Janson, M., Asensio-Torres, R., \& Köhler, R. 2019, A\&A, 627, A167

Close, L. M., Lenzen, R., Guirado, J. C., et al. 2005, Nature, 433, 286

Covey, K. R., Lada, C. J., Román-Zúñiga, C., et al. 2010, ApJ, 722, 971

Cushing, M. C., Rayner, J. T., \& Vacca, W. D. 2005, ApJ, 623, 1115

Cushing, M. C., Marley, M. S., Saumon, D., et al. 2008, ApJ, 678, 1372

Daemgen, S., Siegler, N., Reid, I. N., \& Close, L. M. 2007, ApJ, 654, 558

David, T. J., Hillenbrand, L. A., Gillen, E., et al. 2019, ApJ, 872, 161

Dressing, C. D., \& Charbonneau, D. 2013, ApJ, 767, 95
Duchêne, G., \& Kraus, A. 2013, ARA\&A, 51, 269

Dupuy, T. J., Liu, M. C., Bowler, B. P., et al. 2010, ApJ, 721, 1725

Eisenhauer, F., Abuter, R., Bickert, K., et al. 2003, in Instrument Design and Performance for Optical/Infrared Ground-based Telescopes, Proc. SPIE, 4841

Freudling, W., Romaniello, M., Bramich, D. M., et al. 2013, A\&A, 559, A96

Gagné, J., \& Faherty, J. K. 2018, ApJ, 862, 138

Gagné, J., Lafrenière, D., Doyon, R., Malo, L., \& Artigau, E. 2014a, ApJ, 783, 121

Gagné, J., Lafrenière, D., Doyon, R., Malo, L., \& Artigau, E. 2014b, ApJ, 798,

Gagné, J., Faherty, J. K., Cruz, K. L., et al. 2015, ApJS, 219, 33

Gaia Collaboration (Prusti, T., et al.) 2016, A\&A, 595, A1

Gaia Collaboration (Brown, A. G. A., et al.) 2018, A\&A, 616, A1

Gaidos, E., Mann, A. W., Lépine, S., et al. 2014, MNRAS, 443, 2561

Gillon, M., Jehin, E., Lederer, S. M., et al. 2016, Nature, 533, 221

Goodwin, S. P., Nutter, D., Kroupa, P., Ward-Thompson, D., \& Whitworth, A. P. 2008, A\&A, 477, 823

Gorlova, N. I., Meyer, M. R., Rieke, G. H., \& Liebert, J. 2003, ApJ, 593, 1074

Hillenbrand, L. A., \& White, R. J. 2004, ApJ, 604, 741

Janson, M., Brandner, W., Lenzen, R., et al. 2007, A\&A, 462, 615

Janson, M., Hormuth, F., Bergfors, C., et al. 2012, ApJ, 754, 44

Janson, M., Bergfors, C., Brandner, W., et al. 2014a, ApJ, 789, 102

Janson, M., Bergfors, C., Brandner, W., et al. 2014b, ApJS, 214, 17

Johnson, J. A., Gazak, J. Z., Apps, K., et al. 2012, AJ, 143, 111

Jones, J., White, R. J., Boyajian, T., et al. 2015, ApJ, 813, 58

Kirkpatrick, J. D., Barman, T. S., Burgasser, A. J., et al. 2006, ApJ, 639, 1120

Klutsch, A., Freire Ferrero, R., Guillout, P., et al. 2014, A\&A, 567, A52

Kraus, A. L., \& Hillenbrand, L. A. 2007, AJ, 134, 2340

Köhler, R., Ratzka, T., \& Leinert, C. 2012, A\&A, 541, A29

Malo, L., Doyon, R., Lafrenière, D., et al. 2013, ApJ, 762, 88

Mamajek, E. E., Kenworthy, M. A., Hinz, P. M., \& Meyer, M. R. 2010, AJ, 139, 919

Manara, C. F., Testi, L., Rigliaco, E., et al. 2013, A\&A, 551, A107

Mentuch, E., Brandeker, A., van Kerkwijk, M. H., Jayawardhana, R., \& Hauschildt, P. H. 2008, ApJ, 689, 1127

Mizuki, T., Kuzuhara, M., Mede, K., et al. 2018, ApJ, 865, 152

Montet, B. T., Bowler, B. P., Shkolnik, E. L., et al. 2015, ApJ, 813, L11

Muirhead, P. S., Hamren, K., Schlawin, E., et al. 2012, ApJ, 750, L37

Muirhead, P. S., Becker, J., Feiden, G. A., et al. 2014, ApJS, 213, 5

Rayner, J. T., Cushing, M. C., \& Vacca, W. D. 2009, ApJS, 185, 289

Riaz, B., Gizis, J. E., \& Harvin, J. 2006, AJ, 132, 866

Rodet, L., Bonnefoy, M., Durkan, S., et al. 2018, A\&A, 618, A23

Rojas-Ayala, B., Covey, K. R., Muirhead, P. S., \& Lloyd, J. P. 2012, ApJ, 748, 93

Schlieder, J. E., Bonnefoy, M., Herbst, T. M., et al. 2014, ApJ, 783, 27

Sembach, K. R., \& Savage, B. D. 1992, ApJS, 83, 147

Skrutskie, M. F., Cutri, R. M., Stiening, R., et al. 2006, AJ, 131, 1163

Torres, C. A. O., Quast, G. R., Melo, C. H. F., \& Sterzik, M. F. 2008, Handbook of Star Forming Regions, Volume II: The Southern Sky ASP Monograph Publications, ed. Bo. Reipurth, 5

Zuckerman, B., \& Song, I. 2004, ARA\&A, 42, 685 